\title{
Flame Retardancy of wood treated with GUP by Cone Calorimetry
}

\author{
Ming GAO ${ }^{1, a}$, Yixin $\mathrm{XU}^{2, \mathrm{~b}}$ \\ ${ }^{1}$ School of Environmental Engineering, North China University of Science and Technology, Box \\ 206, Yanjiao Beijing 101601, China \\ ${ }^{2}$ School of Material Science and Engineering, Zhejiang University, Hangzhou 310058, China \\ agaoming@ncist.edu.cn, b382553866@qq.com
}

Key words: cone calorimeter, flame retardant, GUP, wood.

Abstract. Wood was treated with guanyl urea phosphate (GUP) to impart flame retardant. The flame retarding behavior of samples was valued by cone calorimeter and thermalgravimetric analysis. The flammability parameters, including rate of heat release (RHR), total heat release (THR), total mass loss (TML) and mass loss rate (MLR), yield of $\mathrm{CO}$ and $\mathrm{CO}_{2}$, smoke production rate (SPR) and total smoke production (TSP) were recorded simultaneously. By analyzing these data, it was concluded that most combustion parameters of wood were decreased by the treatment.

\section{Introduction}

Wood, which mainly consists of cellulose, lignin and hemicellulose, catches fire easily and burns vigorously with flame. The use of wood in house interiors, building or public transport constitutes a potential hazard for people in case of fire. The need for consumer protection, coupled with the new regulations and environmental concerns, increases the interest in flame-retardant treatments. Illustrative of various past approaches of imparting flame retardant of wood are treatment with compositions containing phosphorus and basic nitrogen compounds such as ammonium phosphate, melamine-phosphoric acid, urea-dicyandiamide-phosphoric acid and other phosphoric amines to accelerate the formation of a carbonized layer on the materials [1,2]. Often formaldehyde is introduced in order to product a phosphate salt of the methyolated guanyl urea to improve the stability and leach resistance of flame retardant [3]. However, there are some disadvantages associated with these aforementioned compositions due to their use of formaldehyde, urea, and their ultimate $\mathrm{pH}$. For example, formaldehyde may not be environmentally acceptable in some instances and urea is both known to be relatively corrosive and hygroscopic when employed in applied compositions at their applied $\mathrm{pH}$ ranges. The cost of the treated wood produced using these compositions is also relatively expensive.

In this work, guanyl urea phosphate (GUP) as flame retardant, is generally environmentally acceptable, relatively low in toxicity, non-corrosive and non-hygroscopic was used into wood. The flame retarding behavior of samples was evaluated by cone calorimeter and thermalgravimetric analysis.

\section{Experimental}

\section{Chemicals}

To prepare the phosphate salt of the guanyl urea (GUP), water was placed within a container and agitated while 25 grams of dicyandiamide were added. 20 milliliters of phosphoric acid (85\%) were subsequently added. The resulting mixture was heated to approximately $100^{\circ} \mathrm{C}$ and held at that 
temperature for approximately 45 minutes. After the mixture was cooled to room temperature, the concentrated GUP solution was obtained, which could be diluted if needed.

\section{Sample Preparation}

Specimens of willow (North of China) were planed timber in which cellulose amounts to about $45 \%$, lignin and hemi-cellulose contents are $32 \%$ and $15 \%$, respectively. The specific gravity was about 0.31 , ash content was $5 \%$, the moisture content was $7 \%$, and volatile matter contents were $6 \%$. Specimens were impregnated with chemicals at $60^{\circ} \mathrm{C}$ for 2 hours under atmospheric pressure, and were dried at $60^{\circ} \mathrm{C}$. The retention (wt \%) was measured for each sample. Sizes of samples were $100 \mathrm{~mm} \mathrm{~L} \mathrm{x} 100 \mathrm{~mm} \mathrm{~W} \times 3 \mathrm{~mm} \mathrm{~T}$ for the cone calorimeter.

\section{Cone Calorimeter}

The test method using the cone calorimeter was ASTM M 1354, Standard Test Method for Heat Release Rates for Materials and Products Using a Consumption Calorimeter. Approximately 13.1 MJ of heat were released per kilogram of oxygen consumed. The external heat fluxes usually chosen were $35 \mathrm{~kW} / \mathrm{m}^{2}$ because it corresponds to a common heat flux in mild fire. Our samples were exposed to a Stanton Redcroft Cone Calorimeter under a heat flux of $35 \mathrm{~kW} / \mathrm{m}^{2}$. The cone data reported in this work were the average of three replicated experiments.

\section{Results and Discussion}

\section{Heat Release}

Rate of heat release (RHR) and total heat release (THR) for different samples are shown in Figures $1-2$, and the corresponding values are listed in Table 1.

Table 1 Data of cone calorimeter

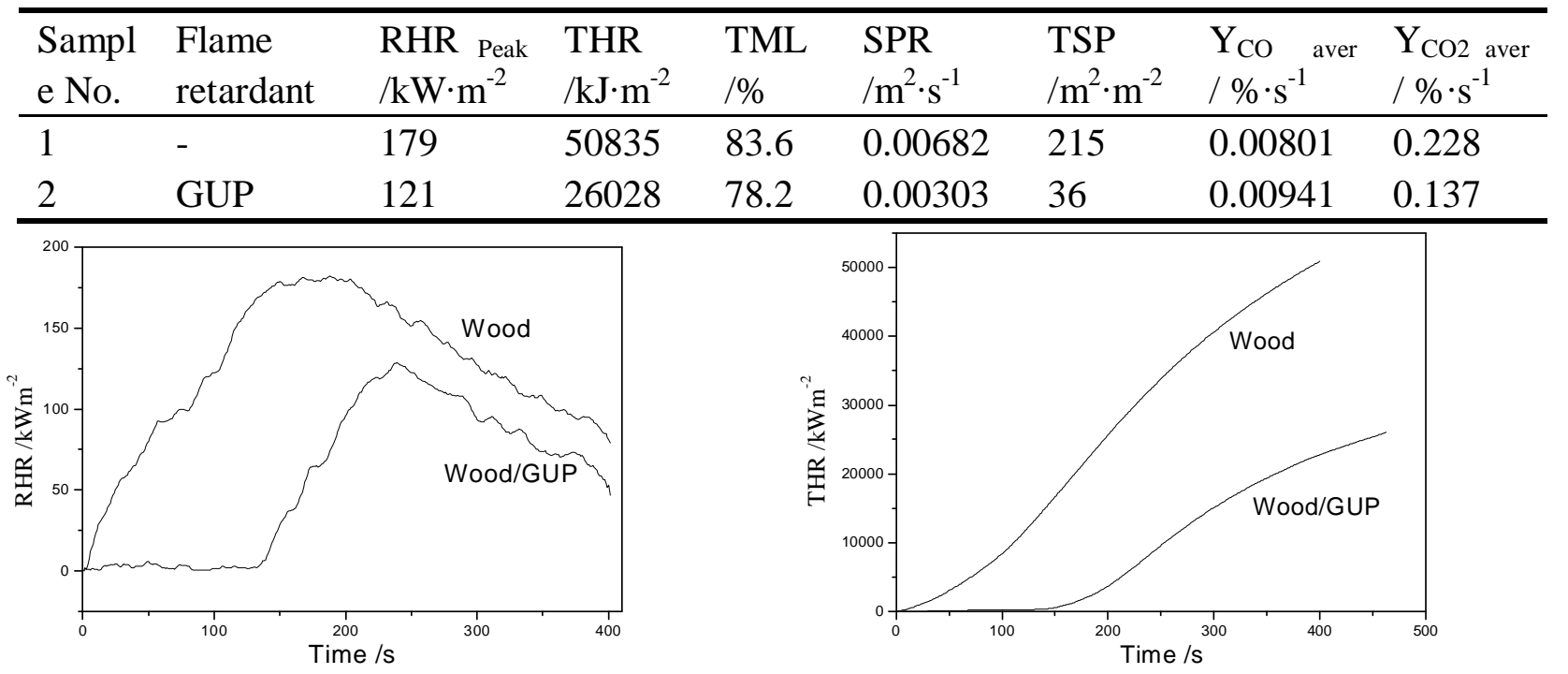

Fig. 1 RHR of the wood samples

Fig. 2 THR of $\mathrm{v}$ the wood samples

From Fig. 1, it is obvious that RHR curves of both virgin wood and wood treated with GUP are not always flat. They all have peaks, which are closely related to their combustion characteristics. According to the reference, there are often three stages of ignition, burning and extinguishment when wood burning. However we can only find two stages in research. This is due to the properties of wood itself.

It is apparent that the flame burning is most important in the growth and fully developed phases of the fire and corresponds to the intensity of the fire. The rate of heat release (RHR) was recognized to quantify the size of fire. From the Fig. 1 and Table.1, it is very clear that a significant flame 
retardant effect is obtained with treatment on wood. The peak of RHR is much decreased by the treatment with flame retardant. Virgin wood shows a RHR peak of $179 \mathrm{~kW} / \mathrm{m}^{2}$ while the RHR peak of wood treated with GUP is $121 \mathrm{~kW} / \mathrm{m}^{2}$. RHR peak is decreased by $32.4 \%$ with GUP. It is because that the samples containing phosphorus can release of phosphoric acid which catalyzes the dehydration and carbonization of wood, resulting in formation of less flammable products and correspondingly more char [4].

From Figure 1, we can also see that there is a platform of RHR values in treated wood rather than virgin wood. Because of it, the peak for treated wood became also delayed for more than 100 seconds. This phenomenon would contribute to more time for heat diffusion when a fire disaster occurs, which means heat cannot be easily concentrated. At last it would decrease the intensity of a fire. This is another factor contributing to flame retardant.

Figure 2 presents the total heat release (THR) for samples. THR means how much heat the materials release during a fire. We expect it would be low. The slope of THR curve can be assumed as representative of fire spread. It is very clear that the flame spread of treated wood is decreased. From the Table 1, we can also see that the total heat release is decreased by the flame retardants. The maximum THR is decreased by $48.8 \%$ with GUP, which suggests there is a remarkable effect of flame retardant in GUP.

\section{Mass Loss}

The total mass loss (TML) and the mass loss rate (MLR) for samples are shown in Figure 4-5, and the corresponding values are also listed in Table 1.

Generally, the function of flame retardant in wood is to increase the char at the cost of flammable volatile products [5]. So the more the char yield is, the better the flame retardant is. The total mass loss (Fig.4, Table 3) is gradually decreasing for treated wood. The total mass loss over the whole test for the virgin wood is $83.6 \%$ of the original mass. For the treated wood higher residues are left. In the case where GUP is used, the total mass loss is $78.2 \%$ of the original mass, decreased by $5.4 \%$, indicating GUP does help to protect wood from burning. Comparing the MLR and RHR curves, they are synchronized in a certain extent. Therefore lower mass loss also means lower heat released.

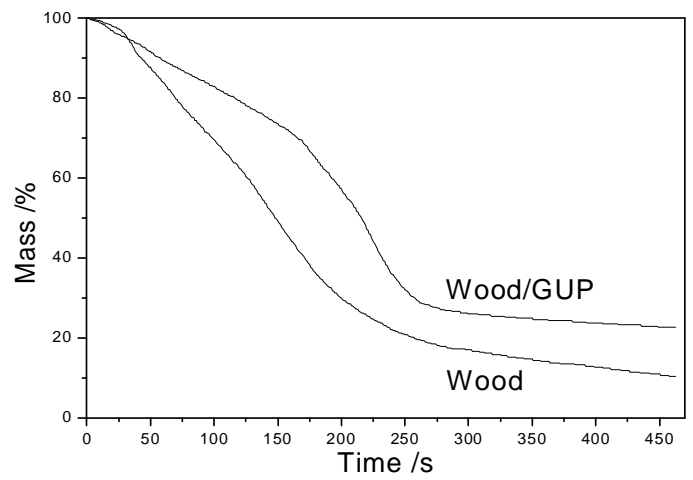

Fig. 3 TML of the wood samples

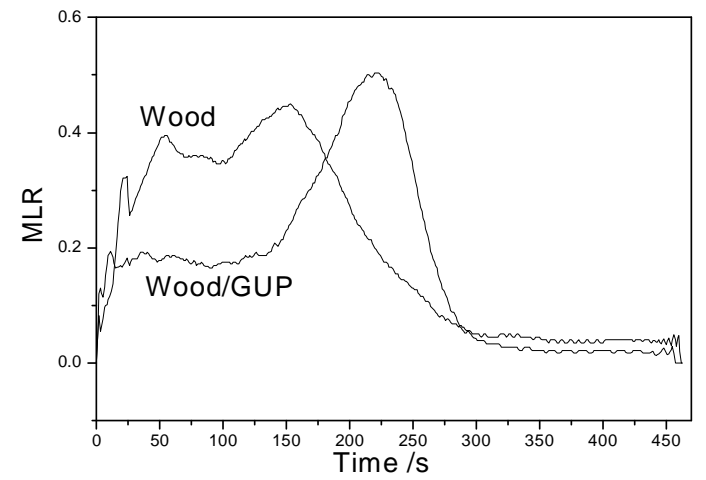

Fig.4 MLR of the wood samples

\section{Gas and Smoke Release}

Carbon monoxide yield $\left(\mathrm{Y}_{\mathrm{CO}}\right)$, carbon dioxide yield $\left(\mathrm{Y}_{\mathrm{CO} 2}\right)$, smoke production rate (SPR) and total smoke production (TSP) for samples are shown in Figure 6-9, and the corresponding values are listed in Table 1. 


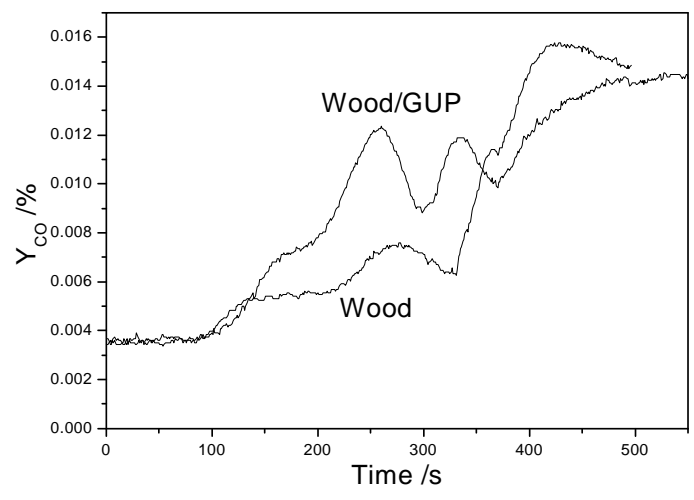

Fig. $5 \mathrm{Y}_{\mathrm{CO}}$ of the wood samples

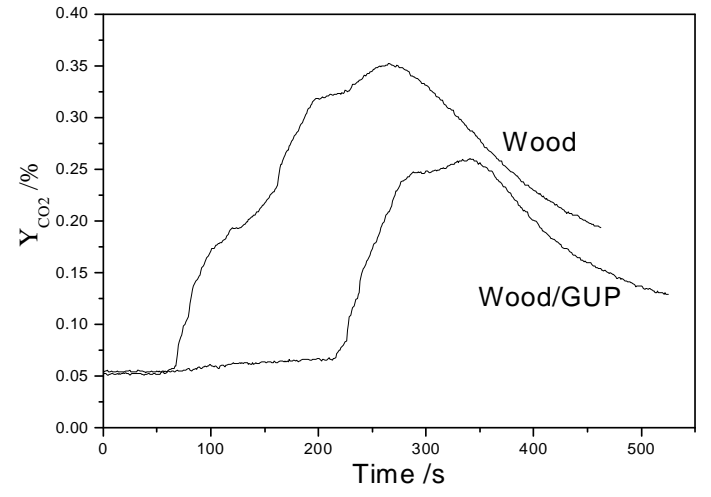

Fig. $6 \mathrm{Y}_{\mathrm{CO} 2}$ of the wood samples

Generally, the smoke production and toxic gas formation along with the heat release rate play a critical role in fire conditions [6]. One of most toxic gases released from burning wood is carbon monoxide. From Fig. 6 and Table 1, we can see that for the flame retardant wood, carbon monoxide yield is relative higher than that of virgin wood. Carbon monoxide is produced in large amount when materials are burned incompletely. Therefore the flame retardant materials often produce more carbon monoxide. The decreasing of $\mathrm{Y}_{\mathrm{CO} 2}$ showed in Fig. 2 also indicates the fact treated samples cannot burn well like virgin wood does. However, the carbon monoxide formation at the expense of carbon dioxide is an important fire retardant principle [6], which means finding methods such as add other synergetic chemicals to inhibit this side effect is also significant.

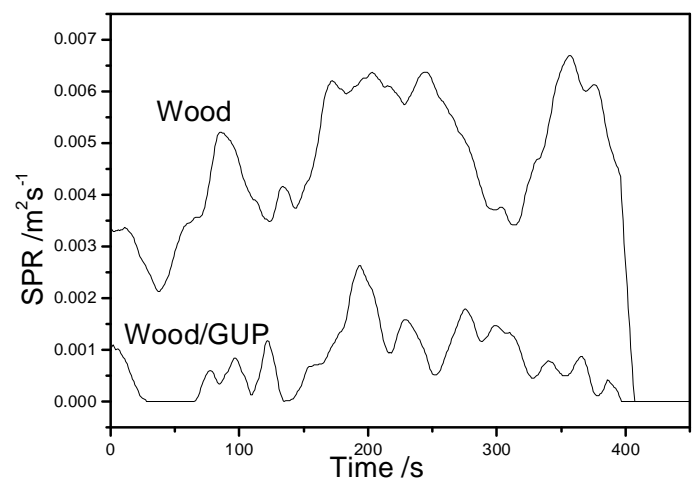

Fig. 7 SPR of the wood samples

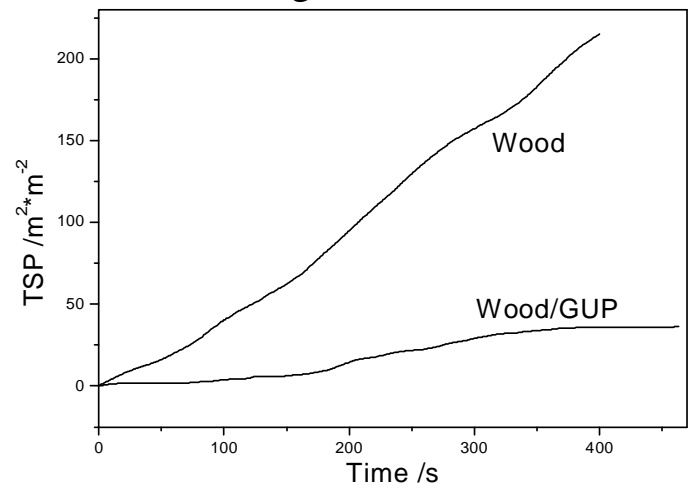

Fig. 8 TSP of the wood samples

The effect of flame retardant on smoke formation was measured. The smoke production rate (SPR) as the function of time is shown in Fig. 7. For samples, smoke is formed during the all burning period. It is obviously that virgin wood has three main peaks. However, the peaks of treated wood are not so obvious. Meanwhile the SPR value is much lower. The SPR maximum of sample with GUP is decreased by $55.5 \%$, illustrating the great smoke inhibiting effect of GUP. Fig. 8 records the total smoke production of all samples. The flame retardant decreases smoke by $83.1 \%$. It also suggests that GUP has great effect on inhibiting smoke, which is meaningful to fire rescuing.

\section{Summary and conclusion}

The flammability parameters of wood treated with GUP, including rate of heat release (RHR), total heat release (THR), total mass loss (TML) and mass loss rate (MLR), smoke production rate (SPR) and total smoke production (TSP) are considerably decreased. GUP can accelerate dehydration and carbonization of wood, which result in more char and less flammable volatile products, obtaining a better flame retardant. However at the same time, there is more CO produced due to flame retardant, which should be solved by other methods. 


\section{Acknowledgement}

The work was supported by the fundamental research funds for the Central Universities (3142015021)

\section{References}

1. H, Getto ; Ishihara, S: Fire Mater. (1998), 22, 77.

2. R. B Kemp: Handbook of Thermal Analysis and Calorimetry, Elsevier Science B. V. Wood, Vol. 4 (1999), Chap.14, Ireland.

3. Loyvet; G. Andre; Morgan; J. Dennis: U. S. Pat. No. 4,461,720, 1984.

4. R. K. Jain, K. Lal, H. L. Bhatnagar: J. Appl. Polym. Sci. 1985, 30, 897.

5. Gao, M.; Sun, C. Y.; Zhu, K. J. Therm. Anal. Cal. 2004, 75, 221.

6. O. Grexa, H. Lubke: Polymer Degradation and Stability, 2001, 74, 427. 